http://jmscr.igmpublication.org/home/ ISSN (e)-2347-176x ISSN (p) 2455-0450 crossref DOI: https://dx.doi.org/10.18535/jmscr/v9i10.14

\title{
Palisading Neutrophilic Granulomatous Dermatitis - A Rare Case Report
}

\author{
Authors \\ Dr Sourabh Sharma ${ }^{1}$, Dr Prajul Mehta ${ }^{2}$, Dr Sakshi Bhota ${ }^{3 *}$ \\ ${ }^{1} \mathrm{MD}$ Dermatology CH Bilaspur, H.P. India \\ ${ }^{2}$ MD Dermatology CH Theog, Shimla, H.P. India \\ ${ }^{3}$ MD Community Medicine, CMO Office, Shimla, H.P. India \\ *Corresponding Author \\ Dr Sakshi Bhota \\ MD Community medicine, CMO Office, Shimla, H.P. India
}

\begin{abstract}
Palisaded neutrophilic and granulomatous dermatitis (PNGD) is a rare dermatological condition, which shows various clinical and histopathological features. A 67-year-old gentleman presented with non healing ulcer on right elbow joint for 6 months. He was on treatment from last 6 months. The physical examination was notable with multiple discrete well defined hyperpigmented lesions all over the body with few of them excoriated, forming ulcers with pus discharge. He also had a non healing ulcer with pus discharge over right elbow joint.

Keywords: PNGD, ulcer, non healing.
\end{abstract}

\section{Introduction}

Palisaded neutrophilic and granulomatous dermatitis (PNGD) is a rare dermatological condition, which shows various clinical and histopathological features. ${ }^{1}$ In 1994, PNGD was proposed to unify the conditions in which immune complex molecules, such as IgM and C3, were present, and a similar distribution pattern of neutrophils was seen upon histologic examination. ${ }^{2}$ PNGD has been reported to be associated with a variety of systemic conditions, such as rheumatoid arthritis (RA), and systemic lupus erythematosus (SLE). Moreover, PNGD has been reported to be associated with antineutrophilic cytoplasmic antibody (ANCA)associated vasculitis, including granulomatosis with polyangiitis (GPA) and microscopic polyangiitis. $^{3-4}$

\section{Case Report}

A 67-year-old gentleman, presented with non healing ulcer on right elbow joint for 6 months. The patient was on treatment from the last 6 months. He was initially evaluated and managed in other hospital. In view of progressive detoriation of clinical status he was referred to us. He was conscious, oriented, febrile (temperature: $100^{\circ} \mathrm{F}$ ) and tachycardia (heart rate: $106 / \mathrm{min}$ ) at the time of presentation.

Physical examination was notable with multiple discrete well defined hyperpigmented lesions all over the body with few of them excoriated, forming ulcers with pus discharge. He also had a non healing ulcer with pus discharge over right elbow joint. Baseline blood investigations revealed anemia (Haemoglobin: $9.30 \mathrm{gm} / \mathrm{dl}$ ), significantly elevated ESR (98 mm/hr) and A: G 
reversal (Albumin: 2.4mg/dl, globulin: $4.6 \mathrm{mg} / \mathrm{dl}$, A: G 0.52).

Blood and tissue samples were sent for culture and sensitivity testing and he was started on empirical antibiotics. Bilateral upper limb arterial and venous doppler did not show any evidence of thrombosis. Wound debridement was done and samples were sent for histopathological examination (HPE).

HPE report shows biopsy tissue covered by keratinized stratified squamous epithelium revealing marked hyperkeratosis, focal parakeratosis, partially superficial crust composed off sheet of neutrophils admix with fibrinous materials. A focus of surface ulceration with adjcent acanthotic epidermis. The superficial dermis revealing infiltration of neutrophils, hystrocytes and lymphocytes intestinal in dermis collagen with focally ecstatic vascular channel and leukocytoclasia. Few scattered neutrophil and lymphocyte are seen intersstrially in the reticular dermis. Focal palisaded appearance of histocytes and stringy mucus are seen. Histological findings were suggestive of palisaded neutrophilic and granulomatous dermatitis. No evidence of malignancy was seen.

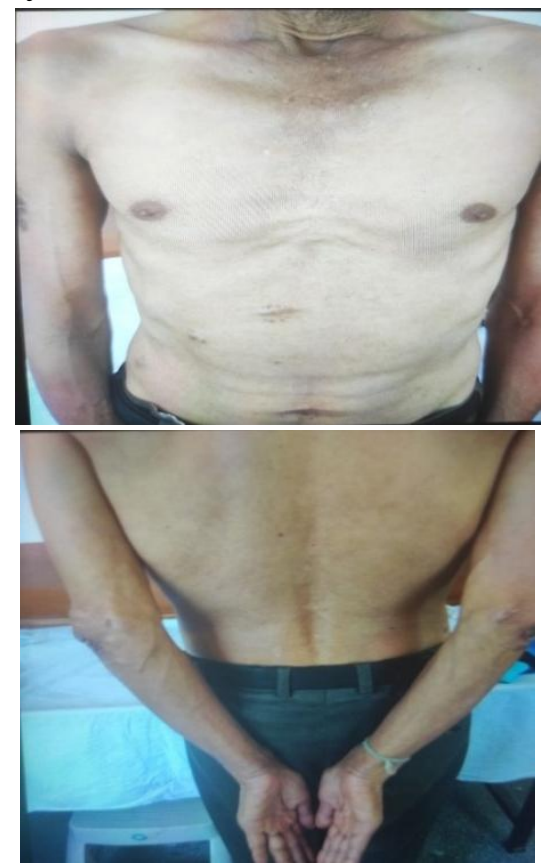

Figure 1\&2 shows multiple discrete well defined hyperpigmented lesions all over the body with few of them excoriated, forming ulcers with pus discharge.

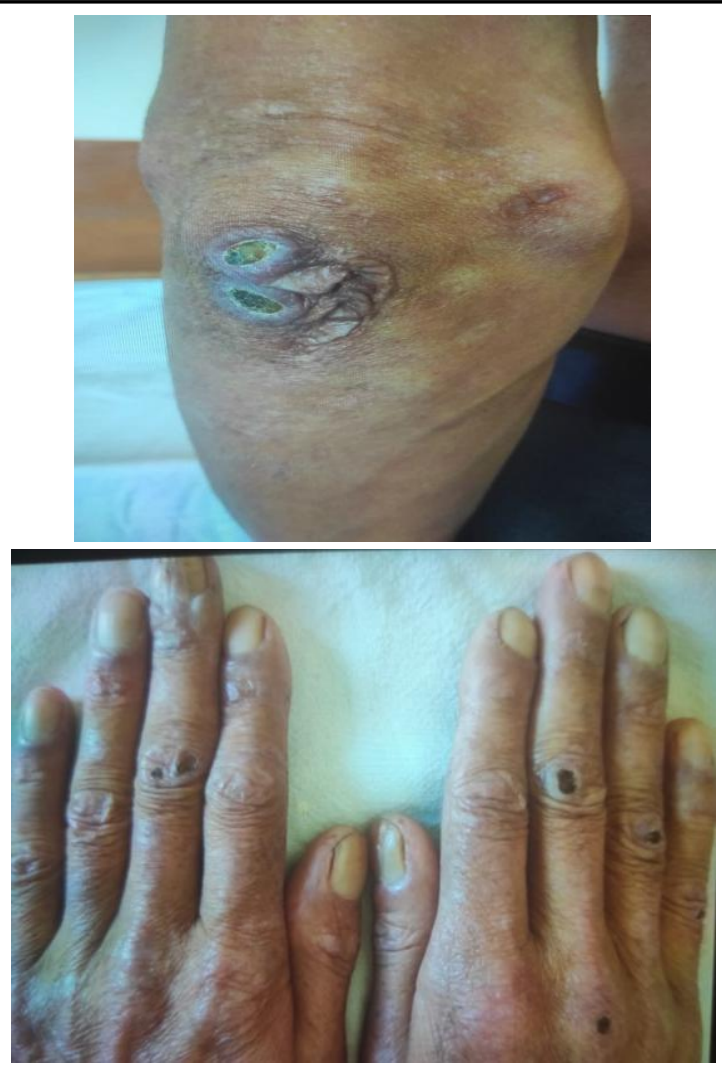

Figure $3 \& 4$ shows non healing ulcer with pus discharge over elbow joint and dorsum of hands.

\section{Discussion}

PNGD and IGD represent two clinicopathologic patterns of granulomatous dermatitis. Some authors use the term "reactive granulomatous dermatitis" instead of using the previous two terms.

PNGD is observed most often in patients with RA, systemic lupus erythematosus, or systemic vasculitis, particularly granulomatosis with polyangiitis (Wegener's granulomatosis and Churg-Strauss syndrome). Other underlying diseases include systemic sclerosis, Sjogren syndrome, autoimmune thyroiditis, hepatitis, inflammatory bowel disease, myelodysplastic syndrome, Takayasu arteritis, diabetes, and infections, such as Streptococcus, HIV, EpsteinBarr virus, and parvovirus. ${ }^{5}$

The etiopathogenesis of PNGD is unknown. The initiating insult in PNGD may involve immune complex deposition around the walls of small dermal blood vessels as a result of the underlying disease, which leads to a subacute or chronic neutrophil-rich small-vessel vasculitis. This in 
turn impairs blood flow to the area; consequently, there is degeneration of the local collagen. The degenerated collagen may incite an immune response, resulting in a palisading lymphohistiocytic infiltrate. This cascade of events is likely aggravated or precipitated by exogenous trauma, which explains the predilection of PNGD lesions for the extensor surfaces. ${ }^{6}$

PNGD is self-limited disease in $20 \%$ of the cases. Although, in the presented case, PNGD skin lesions improved after high potency topical steroid and oral colchicine treatment, we could not conclude whether improvement was due to the drugs or spontaneous resolution of the lesions. ${ }^{7}$

Early lesions of PNGD show a dense neutrophilic infiltration with or without cutaneous small-vessel vasculitis (leukocytoclastic vasculitis), and collagen degeneration, and lesions are clinically similar with erythematous macules or urticarialike annular plaques with crusting. Fully developed lesions are characterized by palisaded granulomas surrounding degenerated collagen with interstitial fibrin and mucin deposits, sparse neutrophils, and nuclear dust that can be visualized through histological examination. Furthermore, the resolving lesions show palisaded granulomas with dermal fibrosis and rare neutrophilic debris. ${ }^{8}$

\section{Conclusion}

PNGD is rare dermatoses of unknown aetiology. It has been described to be associated with a number of autoimmune conditions and drugs. It is very important for clinicians to have knowledge about this rare skin condition as it may be heralding presentation of a serious underlying condition like lymphoproliferative disorders or solid organ malignancies. There is no specific treatment and causative disease has to be diagnosed and targeted. It may resolve spontaneously with control of primary disease. The disease course is characterized by periods of flares and remissions.

\section{Declaration of Patient Consent}

The authors certify that they have obtained all appropriate patient consent forms. In the form the patient(s) has/have given his/her/ their consent for his/her/their images and other clinical information to be reported in the journal. The patients understand that their names and initials will not be published and due efforts will be made to conceal their identity, but anonymity cannot be guaranteed.

\section{Conflict of Interest- Nil}

\section{References}

1. Misago N, Shinoda Y, Tago M, Narisawa Y. Palisaded neutrophilic granulomatous dermatitis with leukocytoclastic vasculitis in a patient without any underlying systemic disease detected to date. J Cutan Pathol. 2010 Oct;37(10):1092-7.

2. Chu P, Connolly MK, LeBoit PE. The histopathologic spectrum of palisaded neutrophilic and granulomatous dermatitis in patients with collagen vascular disease. Arch Dermatol. 1994 Oct;130(10):127883.

3. Sangueza OP, Caudell MD, Mengesha YM, Davis LS, Barnes CJ, Griffin JE, et al. Palisaded neutrophilic granulomatous dermatitis in rheumatoid arthritis. J Am Acad Dermatol. 2002 Aug;47(2):251-7.

4. Maurelli M, Colato C, Girolomoni G. Palisaded neutrophilic granulomatous dermatitis and its associations with autoimmune diseases. Eur J Dermatol. 2019 Aug; 29(4):432-3.

5. Piette WW. Rheumatoid arthritis, juvenile idiopathic arthritis, adultonset still disease, and rheumatic fever. In: Kang S, Amagai M, Bruckner AL, Enk AH, Margolis DJ, McMichael AJ,et al., Fitzpatrick's Dermatology. 9th ed. Vol. 1. New York: McGraw Hill; 2019. p. 1148-9

6. Kalen JE, Shokeen D, Ramos-Caro F, Motaparthi K. Palisaded neutrophilic 
granulomatous dermatitis: Spectrum of histologic findings in a single patient. JAAD Case Rep 2017;3:425-8.

7. Mahmoodi M, Ahmad A, Bansal C, Cusack CA. Palisaded neutrophilic and granulomatous dermatitis in association with sarcoidosis. J Cutan Pathol 2011;38:365-8.

8. Finan MC, Winkelmann RK. The cutaneous extravascular necrotizing granuloma (Churg-Strauss granuloma) and systemic disease: a review of 27 cases. Medicine (Baltimore) 1983;62:142-58. 